\title{
High-grade gliomas in survivors of childhood acute lymphoblastic leukaemia
}

\author{
George A. Alexiou
}

Received: 23 March 2009/Published online: 19 May 2009

(C) Springer-Verlag 2009

\section{Dear Editor:}

We read with great interest the recent article by Bien et al. on high-grade gliomas that occurred in children cured of acute lymphoblastic leukaemia (ALL). In this study, the authors reported two cases of glioblastoma multiforme and one case of anaplastic astrocytoma with poor overall survival despite aggressive treatment. Cranial irradiation has been considered as the most important factor for the development of secondary brain tumours. The authors concluded that early detection followed by complete resection and chemoradiotherapy is of paramount importance; however, they do not warrant better prognosis [1].

We recently studied the secondary malignant brain tumours in children who were previously treated for ALL in our institute and we encountered one case of glioblastoma multiforme and two cases of anaplastic oligodendrogliomas. The tumours developed after a mean period of 6.8 years after ALL treatment. The occurrence of anaplastic oligodendrogliomas is exceedingly rare and only eight cases have been reported in the literature. For further investigation, we undertook a molecular cytogenetic study of the oligodendroglioma specimens obtained from the two patients using fluorescence in situ hybridization (FISH) with DNA probes mapping to chromosome 1p36, 19p13 and 19q13. FISH analysis demonstrated in both cases $1 \mathrm{p} 36$ deletions (unpublished data). Studies of oligodendroglioma patients indicated that a loss of chromosome $1 p$ and $19 q$ was associated with high sensitivity to chemotherapy and radiotherapy and a better probability of survival [2]. We believe that it would be interesting to investigate if the allelic loss differs from those observed in primary lesions. Further research is needed to clarify if certain underlying genetic characteristics may also be associated with an increased risk of radiation-induced brain tumours.

\section{References}

1. Bien E, Stachowicz-Stencel T, Szalewska M, Bien E, StachowiczStencel T, Szalewska M, Krawczyk M, Synakiewicz A, Dubaniewicz-Wybieralska M, Zielinski P, AdamkiewiczDrozynska E, Balcerska A (2009) Poor-risk high-grade gliomas in three survivors of childhood acute lymphoblastic leukaemia - an overview of causative factors and possible therapeutic options. Childs Nerv Syst 25:619-626

2. Smith JS, Perry A, Borell TJ, Lee HK, O'Fallon J, Hosek SM, Kimmel D, Yates A, Burger PC, Scheithauer BW, Jenkins RB (2000) Alterations of chromosome arms $1 p$ and $19 q$ as predictors of survival in oligodendrogliomas, astrocytomas, and mixed oligoastrocytomas. J Clin Oncol 18:636-645
G. A. Alexiou

Department of Neurosurgery, Children's Hospital "Agia Sofia",

Athens, Greece

G. A. Alexiou $(\bowtie)$

Aetideon 52,

Cholargos,

Athens 15561, Greece

e-mail: alexiougrg@yahoo.gr

e-mail: alexiougr@gmail.com 